\title{
A retarded coupling approach to intermolecular interactions
}

\author{
L C Dávila Romero ${ }^{1}$ and D L Andrews \\ School of Chemical Sciences, University of East Anglia, Norwich NR4 7TJ, UK \\ E-mail: 1.davila-romero@uea.ac.uk
}

Received 12 December 2008, in final form 12 March 2009

Published 9 April 2009

Online at stacks.iop.org/JPhysB/42/085403

\begin{abstract}
A wide range of physical phenomena such as optical binding and resonance energy transfer involve electronic coupling between adjacent molecules. A quantum electrodynamical description of these intermolecular interactions reveals the presence of retardation effects. The clarity of the procedure associated with the construction of the quantum amplitudes and the precision of the ensuing results for observable energies and rates are widely acknowledged. However, the length and complexity of the derivations involved in such quantum electrodynamical descriptions increase rapidly with the order of the process under study. Whether through the use of time-ordering approaches, or the more expedient state-sequence method, time-consuming calculations cannot usually be bypassed. A simple and succinct method is now presented, which provides for a direct and still entirely rigorous determination of the quantum electrodynamical amplitudes for processes of arbitrarily high order. Using the approach, new results for optical binding in two- and three-particle systems are secured and discussed.
\end{abstract}

\section{Introduction}

A wide range of physical phenomena involve electronic coupling between adjacent molecules [1-7]. In connection with optical binding, for example, the interactions of neighbouring static electric and induced dipoles produce the potential energy landscapes that determine local forces and positions of equilibrium [8,9], while the dynamic interactions between transition dipoles are responsible for intermolecular energy transfer [10-12]—the latter itself a process that engenders modifications to the local force fields [13-15]. When off-resonant radiation propagates through a molecular system it engages further pair interactions that are reflected in optical binding forces $[16,17]$ and modifications to the rates of energy transfer [18].

At a fundamental level, the theory of all such interactions is deliverable on a quantum electrodynamical basis, some of its main advantages over other methods being rigour and the automatic inclusion of retardation effects [16, 19]. The latter can prove surprisingly prominent, as for example in the progression of resonance energy transfer from a radiationless to a radiative character as the intermolecular distance increases $[10,11]$. The clarity of procedure associated with the

\footnotetext{
1 Author to whom correspondence should be addressed.
}

construction of quantum electrodynamical amplitudes and the precision of the ensuing results for observable energies and rates are widely acknowledged. However, it is the case that the complexity and length of these calculations become progressively more daunting as the order of the process increases. For example, calculation of the well-known Casimir-Polder potential, which is of fourth order in electric dipole interactions, requires the consideration of 24 different time orderings, each associated with a Feynman graph and each contributing differently to the result [20, 21]. Similar or higher degrees of complexity arise in the calculation of optical binding potentials, and a variety of other processes involving molecular pairs [22, 23]. When three particles are involved, the number of contributions escalates hugely, and the algebra involved is not at all amenable to implementation with conventional software [24].

One expedient means of tackling the problem of calculational complexity of high-order intermolecular interactions is a method based on state sequences rather than time orderings [25]. A rigorous basis for the associated diagrammatic constructions has been worked out and the calculational procedures detailed; various applications including the Casimir-Polder interaction have recently been undertaken [21]. One advantage over the 
Feynman diagrammatic basis is that, for a given process, a single state-sequence diagram concisely accommodates the same information that would be cast in a complete set of Feynman diagrams - in one typical case 720 time orderings are built into a single, compact state-sequence graph. Another advantage of the state-sequence representation is that it identifies intermediate virtual states that are not apparent from the Feynman approach. Yet, despite the advantages in setting up and performing the necessary calculations, considerable complexity cannot be circumvented-which is true whatever traditional construct of theory is deployed.

Against this background, we report a new and rigorous algorithm-based method that allows direct determination of the quantum electrodynamical amplitudes for processes of arbitrarily high order. No approximations are involved; the ensuing results are precisely those that would also emerge from the much lengthier methods of calculation outlined above. The following account details the basis for the procedure and the means of its implementation; applications to several illustrative processes are also given, including resonance energy transfer and its optical (laser-assisted) control, and optical pair interactions. Using the approach, new results for optical binding in two- and three-particle systems are secured and discussed.

\section{Theoretical description}

Let us consider a molecular system formed by multiple centres. Using the generic term 'centres', we mean to accommodate systems composed of single molecules, as well as molecular clusters, micro- or nano-particles; for succinctness, we will refer to them as either molecules or centres, unless otherwise stipulated. The centres are interacting through coupling with electromagnetic fields, either assisted by throughput radiation or, as in the case of static coupling or dispersion interactions, through the electromagnetic vacuum, see below [26, 27]. The centres, labelled $\xi$, are electronically distinct and neutral. The closed system can be described by a Hamiltonian $H$ written as a sum of three terms: (i) the second-quantized Hamiltonian for radiation field in vacuo, $H_{\text {rad }}$; (ii) a sum over all molecular centres of the corresponding non-relativistic Hamiltonians, $H_{\text {mol }}(\xi)$; (iii) a similar sum of the interaction Hamiltonians that address the coupling between each centre and the radiation field, $H_{\text {int }}(\xi)[20,28]$ :

$$
H=H_{\mathrm{rad}}+\sum_{\xi} H_{\mathrm{mol}}(\xi)+\sum_{\xi} H_{\mathrm{int}}(\xi) .
$$

Each of the latter interaction terms $H_{\text {int }}(\xi)$ can be expressed as a sum of multipolar contributions. Using the convention of implied summation over repeated indices, we have

$$
\begin{gathered}
H_{\mathrm{int}}(\xi)=-\varepsilon_{o}^{-1} \mu_{i}(\xi) d_{i}^{\perp}\left(\mathbf{R}_{\xi}\right)-m_{i}(\xi) b_{i}\left(\mathbf{R}_{\xi}\right) \\
-\varepsilon_{o}^{-1} Q_{i j}(\xi) \cdot \nabla_{j} d_{i}^{\perp}\left(\mathbf{R}_{\xi}\right)-\cdots .
\end{gathered}
$$

where $\mu_{i}(\xi), m_{i}(\xi)$ and $Q_{i j}(\xi)$ are the electric-dipole, magnetic-dipole and electric-quadrupole tensors, respectively. The interaction Hamiltonian $H_{\text {int }}(\xi)$ also depends on the transverse electric field operator, $d_{i}^{\perp}\left(\mathbf{R}_{\xi}\right)$, and the magnetic field operator, $b_{i}\left(\mathbf{R}_{\xi}\right)$. In practice, the first term of (2), representing electric-dipole interactions, often suffices. For example, in systems where the radiative wavelengths involved are much longer than the dimensions of the centres $\xi$, the field strength may be considered as uniform and its gradient, with which quadrupoles and higher multipoles engage, essentially zero. However, for other processes such as optical activity, it is necessary to include magnetic and higher -order electric multipole contributions [20,29]. In general, it is required to consider only the form of coupling affected by the lowest order of electric multipole that supports each inter-particle transition. Moreover, a traceless form can be adopted for the quadrupole and higher order multipoles, consistent with the divergencefree character of the electric displacement field [30]. In this work we shall limit our studies to the electric multipolar interactions, referred to by the shorthand notation $\mathrm{E} n$ for the multipolar interaction of order $n$. If required, incorporation of the magnetic counterparts $\mathrm{M} n$ is straightforward.

Within the multipolar interaction framework, all intermolecular interactions are mediated by the exchange of transverse photons. The multipolar form can be derived by use of the Power-Zienau-Woolley (PZW) canonical transformation from the also well-known minimal coupling representation [20, 28, 31]. Through the use of perturbation theory, the generalized expression for the coupling between two electric multipoles Em-En can simply be written as $[32,33]$

$$
M_{f i}^{\mathrm{E} m-\mathrm{E} n}=E_{A i_{1} \ldots i_{m}}^{(m)} V_{i_{1} \ldots i_{m} j_{1} \ldots j_{n}}(\kappa, \mathbf{R}) E_{B j_{1} \ldots j_{n}}^{(n)},
$$

where $E_{\xi}^{(m)}$ is the transition multipole tensor of order $m$ for centre $\xi$. If three centres are involved, such as in [24, 34], the coupling takes the form

$$
\begin{aligned}
& M_{f i}^{\mathrm{E} m-\mathrm{E} p-\mathrm{E} q}=E_{A i_{1} \ldots i_{m}}^{(m)} V_{i_{1} \ldots i_{m} j_{1} \ldots j_{n}}\left(\kappa, \mathbf{R}_{B A}\right) \\
& \quad \times E_{B j_{1} \ldots j_{n} k_{1} \ldots k_{p-n}}^{(p)} V_{k_{1} \ldots k_{p-n} l_{1} \ldots j_{q}}\left(\kappa, \mathbf{R}_{C B}\right) E_{C l_{1} \ldots l_{q}}^{(q)},
\end{aligned}
$$

where $\mathbf{R}_{\xi \xi^{\prime}}=\mathbf{R}_{\xi}-\mathbf{R}_{\xi^{\prime}}$. The coupling tensor, $V_{i_{1} \ldots i_{m} j_{1} \ldots j_{n}}(\kappa, \mathbf{R})$, quantifying the retarded interaction between two centres, signifies the propensity for any Em emission and/or absorption process at $A$ to be electromagnetically registered at $B$ and vice versa. Its explicit result, which involves lengthy calculations based on summing contributions of all time orderings (see for example [12]), is

$$
\begin{aligned}
& V_{i_{1} \ldots i_{m} j_{1} \ldots j_{n}}(\kappa, R)=\frac{(-1)^{m}}{4 \pi \varepsilon_{0}} \nabla_{i_{2}} \ldots \nabla_{i_{m}} \nabla_{j_{2}} \ldots \nabla_{j_{n}} \\
& \times\left(-\nabla^{2} \delta_{i_{1} j_{1}}+\nabla_{i_{1}} \nabla_{j_{1}}\right) \frac{\mathrm{e}^{\mathrm{i} \kappa R}}{R} .
\end{aligned}
$$

The coupling tensor depends on $\kappa$, the magnitude of a generalized wave vector defined according to the optical process under study, and on $\mathbf{R}$, the displacement vector between the two centres linked by the coupling tensor.

The simplest case that may be considered is resonance energy transfer, a process involving the dynamic interaction between a centre $A$ initially in an excited state $\alpha$ and a centre $B$ in its ground state $[10,11,18]$. The transfer of energy, involving a coupling of electric-dipole transitions, results in the decay of centre $A$ to its ground state and the excitation of centre $B$ to an excited state $\beta$. From the general expression 
(4) it is readily verified that this E1-E1 dipole coupling [35] is given by

$$
M_{f i}^{\mathrm{E} 1-\mathrm{E} 1}=\mu_{i}^{0 \alpha(\mathrm{A})} V_{i j}(\kappa, R) \mu_{j}^{\beta 0(B)},
$$

where $\kappa=E_{\alpha 0}^{A} / \hbar c$. Here the coupling tensor takes the explicit form

$$
\begin{aligned}
& V_{i j}(\kappa, \mathbf{R})=\frac{\mathrm{e}^{\mathrm{i} \kappa R}}{4 \pi \varepsilon_{0} R^{3}} \\
& \quad \times\left\{(1-\mathrm{i} \kappa R)\left(\delta_{i j}-3 \hat{R}_{i} \hat{R}_{j}\right)-(\kappa R)^{2}\left(\delta_{i j}-\hat{R}_{i} \hat{R}_{j}\right)\right\} .
\end{aligned}
$$

A more detailed description of this process is given in section 4.1. If quadrupole contributions are considered, say in centre $A$, then the quantum amplitude for a mechanism involving the E2-E1 coupling [36] is given by

$$
M_{f i}^{\mathrm{E} 2-\mathrm{E} 1}=Q_{i j}^{0 \alpha(A)} V_{i j k}(\kappa, \mathbf{R}) \mu_{k}^{\beta 0(B)},
$$

involving a rank-3 retarded potential defined as

$$
\begin{aligned}
& V_{i j k}(\kappa, \mathbf{R})=\frac{-\mathrm{e}^{\mathrm{i} \kappa R}}{4 \pi \varepsilon_{0} R^{4}}\left[( 3 - 3 \mathrm { i } \kappa R - \kappa ^ { 2 } R ^ { 2 } ) \left(\delta_{i j} \hat{R}_{k}+\delta_{j k} \hat{R}_{i}\right.\right. \\
& \left.\left.\quad+\delta_{i k} \hat{R}_{j}-5 \hat{R}_{i} \hat{R}_{j} \hat{R}_{k}\right)-\left(\kappa^{2} R^{2}-\mathrm{i} \kappa^{3} R^{3}\right)\left(\delta_{i j}-\hat{R}_{i} \hat{R}_{j}\right) \hat{R}_{k}\right] .
\end{aligned}
$$

In the following sections, we shall focus discussion on electricdipole interactions noting, however, that the incorporation of quadrupole and/or higher multipole interactions is straightforward and intuitive.

Additional insight into the general structure of the results that emerge from detailed QED calculations is afforded by considering a mechanism whereby energy transfer between two centres is influenced by coupling to a third particle, $C$. In a process where the overall effect remains a transfer of excitation from $A$ to $B$, one of the three possible mechanisms involving the third centre is given by the following result in the case of E1-(E1) ${ }^{2}-\mathrm{E} 1$ coupling [24]:

$$
\begin{aligned}
& M_{f i}^{\mathrm{E} 1-(\mathrm{E} 1)^{2}-\mathrm{E} 1}=\mu_{i}^{0 \alpha(A)} V_{i j}\left(\kappa, \mathbf{R}_{C A}\right) \alpha_{j k}^{00(C)}(-\kappa, \kappa) \\
& \quad \times V_{k l}\left(\kappa, \mathbf{R}_{B C}\right) \mu_{l}^{\beta 0(B)},
\end{aligned}
$$

where $\alpha_{j k}^{00(C)}(-\kappa, \kappa)$ is the polarizability tensor of the centre $C$. An explicit expression of this polarizability tensor is given in section 4 , where it arises in the context of a more detailed study of resonance energy transfer within the framework of the retarded coupling method developed below.

From the examples shown in expressions (6), (8) and (10) it can be recognized that the coupling expression for different processes has a generic format involving the contraction of transition multipole tensors $E_{\xi}^{(m)}$ with a coupling tensor $V_{i_{1} \ldots i_{m} j_{1} \ldots j_{n}}(k, \mathbf{R})$. Although there are clearly common features in the analysis of all the above processes, each of the corresponding transition matrices involves a separate derivation. For each individual process, calculation entails either the use of Feynman graphs where all time orderings of intervening interactions are considered [20], or the state-sequence method [25] where such time orderings are accommodated in state-sequence diagrams. Although the latter method offers a more direct calculational route to the result, both methods are taxing. Nevertheless, the examples given in equations (6), (8) and (10) suggest the enticing prospect of the development of a theoretical framework that can embody and exploit the connection between all these processes. For example, it can easily be seen that the transition matrix of say $M$ centres will involve $(M-1)$ coupling tensors, connecting with the molecular tensors of each centre. Thus, in a two-centre process, such as in expressions (6) and (10), the transition matrix $M_{f i}$ has a structure in which two molecular tensors, one for each centre $A$ and $B$, are linked by a third, the coupling tensor $V_{i_{1} \ldots i_{m} j_{1} \ldots j_{n}}(k, \mathbf{R})$. In the case of a three-centre process the transition matrix involves the molecular tensor for each of the three centres, connected by the engagement of two coupling tensors as shown in (8), and so on.

In the following section, we introduce a pragmatic approach to finding an expression that can represent the coupling of intermolecular interactions, based on the molecular tensors of isolated molecules and their mutual contractions with the coupling tensor (equation (7)).

\section{Retarded coupling method}

Equations (6), (8) and (10) are indicative and representative of a general structure in terms of which the quantum amplitudes for multi-centre interactions are cast. The multipole coupling within a system comprising $N$ particles can schematically be represented as

$M_{f i}=R_{\mathrm{rad}} \times \operatorname{Re}\left[\tilde{\chi}_{\left\{k_{1}\right\}}^{\xi_{1}} \times \prod_{j=2}^{N}\left(V_{\left\{k_{j}\right\}\left\{k_{j+1}\right\}}\left(\kappa, \mathbf{R}_{\xi_{j+1} \xi_{j}}\right) \times \tilde{\chi}_{\left\{k_{j}\right\}}^{\xi_{j}}\right)\right]$,

where $R_{\text {rad }}$, defined below, is a scalar factor deriving from the properties of the throughput radiation. Physically, equation (11) signifies that each participant molecule, engaging in the net interaction through its tensor $\tilde{\chi}$ (also defined below), thereby couples to the radiation field, and to other molecules through the retarded potential. The set $\left\{k_{j}\right\}$ represents a set of indices determined by the number of photonic interactions in a particular centre. To find precise expressions for each of the factors and tensors in (11), for any specific intermolecular process, a few simple steps must be followed. It suffices to base our analysis on any one of the time-ordered diagrams (or state-sequence paths) describing it-bearing in mind that either representation is cast in terms of energy non-conserving intermediate states. Thus, although any such time ordering or state sequence can be selected as a basis for the method described below, simplicity is retained by use of the instance whose every stage adheres most closely to energy conservation. (For example, in a scattering process, that instance of time ordering would be the case where photon absorption precedes photon creation.) Restricting our studies to the electric-dipole approximation, we have the following.

(i) The number of interactions (due to virtual or real photons) in each centre $\xi$ determines the molecular tensor rank (in the trivial case with only one interaction present, such a tensor is of rank 1, i.e. the transition dipole moment vector $\left.\mu_{i}^{\xi}\right)$ 
(ii) The radiative scalar factor, $R_{\text {rad }}$, takes the general form [20]

$$
\begin{aligned}
R_{\mathrm{rad}}= & (-\mathrm{i})^{n} \frac{(\hbar c)^{n / 2}\left(k_{1} k_{2} \ldots k_{n}\right)^{1 / 2}\left(n_{1} n_{2} \ldots n_{N}\right)^{1 / 2}}{\left(2 V \varepsilon_{o}\right)^{n / 2}} \\
& \times \exp \left[\mathrm{i} \sum_{i, \xi} \mathbf{k}_{i}^{(\xi)} \cdot \mathbf{R}_{\xi}\right]
\end{aligned}
$$

where it has been assumed that all photonic modes involved in the optical process are different. In cases where two or more interactions with photons of the same mode occur, the degree of coherence of the radiative quantum states, given by $\left(n_{i}\right)^{1 / 2}$ for each interaction, must be replaced accordingly. For example, if emission of two photons of the same mode $n_{i}\left(\mathbf{k}_{i}, \lambda_{i}\right)$ occurs, then the factor would be $\left(n_{i}\left(n_{i}-1\right)\right)^{1 / 2}$ (see also [20]).

(iii) In its explicit and generic form, the $\tilde{\chi}$ tensor for centre $\xi$ identifies with the more commonly known nonlinear hyperpolarizability tensor $\chi_{i_{1} \ldots i_{n}}^{\{\alpha\}\{\xi\}}$ contracted with the polarization vectors of the real photonic modes involved in the process:

$$
\begin{aligned}
& \tilde{\chi}_{\left\{k_{1}\right\}}^{\xi_{1}}=e_{i_{1}}^{\left(\lambda_{1}\right)}\left(\mathbf{k}_{1}\right) \ldots e_{i_{p}}^{\left(\lambda_{n}\right)}\left(\mathbf{k}_{n}\right) \\
& \times \chi_{i_{1} \ldots i_{p}, i_{p+1} \ldots i_{p+n}}^{\{\alpha\}\{s\}(\xi)}\left(\eta_{1} \kappa_{1}, \eta_{2} \kappa_{2}, \ldots, \eta_{N-1} \kappa_{N-1}\right) .
\end{aligned}
$$

Similarities between the retarded coupling method and the induced moment method [37, 38] can then be drawn. The $\tilde{\chi}$ tensor can be related to the same role played by an $n$ th-order-induced electric multipole moment as defined in [39-43], exhibiting a mathematical isomorphism in the results. Moreover, the 'hyperpolarizability' tensor $\chi_{i_{1} \ldots i_{n}}^{\{\alpha\}\{\xi)}$, in equation (13), is the same as is used in onecentre interactions $[20,44]$ :

$$
\begin{aligned}
& \chi_{i_{1} \ldots i_{n}}^{\{\alpha\}\{s\}(\xi)}\left(\eta_{1} \kappa_{1}, \eta_{2} \kappa_{2}, \ldots, \eta_{N-1} \kappa_{N-1}\right) \\
& =\sum_{\pi\{\alpha\},\{s\}}\left\{\mu_{m s_{N-1}}^{\alpha_{n}} \mu_{s_{N-1} s_{N-2}}^{\alpha_{n-1}} \ldots \mu_{s_{2} s_{1}}^{\alpha_{2}} \mu_{s_{1} n}^{\alpha_{1}}\right\} \\
& \quad \times\left\{\left(E_{n s_{1}}-\hbar \eta_{1} c \kappa_{1}\right)\left(E_{n s_{2}}-\hbar \eta_{1} c \kappa_{1}-\hbar \eta_{2} c \kappa_{2}\right) \ldots\right. \\
& \left.\quad \times\left(E_{n s_{N-1}}-\sum_{p=1}^{N-1} \hbar \eta_{p} c \kappa_{p}\right)\right\}^{-1} .
\end{aligned}
$$

However, care must be taken when constructing this tensor for processes involving multi-centre physics. As for single-centre systems, all real photons interacting directly with centre $\xi$ must be considered-but in addition, all other real photons interacting with centres $\xi^{\prime}$, different from $\xi$, also contribute to the molecular tensor $\chi_{i_{1} \ldots i_{n}}^{\{\alpha\}\langle\xi)}$. In other words, all real photons engaged in the overall interaction are represented in the construction of each molecular tensor. The energy denominator factors, for the molecular tensor in (14), also require careful construction. For each centre $\xi$, it is possible to define an effective wave-vector magnitude $\kappa_{i}$, in terms of the magnitudes of the wave vectors of real photons interacting with other centres $\xi^{\prime}$, and their molecular transition energies, $E_{m n}^{\left(\xi^{\prime}\right)}$, such that

$$
\kappa_{i}^{(\xi)}=\sum_{\xi^{\prime}}\left[\frac{E_{m n}^{\left(\xi^{\prime}\right)}}{\hbar c}+\sum_{s}{ }^{\left(\xi^{\prime}\right)} \eta_{s} k_{s}\right] .
$$

(iv) The retarded potential tensor is given by the usual expression (5), $V_{\left\{k_{j}\right\}\left\{k_{j+1}\right\}}\left(\kappa, \mathbf{R}_{\xi_{j+1} \xi_{j}}\right)$, where its parametric dependence is on the effective wave-vector magnitude $\kappa$ as given by (15), while $\mathbf{R}_{\xi_{j+1} \xi_{j}}$ is the separation of the two centres linked by the retarded potential.

\section{Examples and applications}

In this section we review several currently well-studied intermolecular interactions, and we consider them within our new approach. The expression for the transition matrix in each case is obtained by use of the method described in the previous section alone, and compared to previous results. In the following, attention is first focused upon resonance energy transfer (RET), then the considerably more complex case of a laser-assisted counterpart to RET, and thirdly the influence of a third body on such optical phenomena. We then study cases of optical binding for two and three particles. The latter, three-particle case, here entertained in detail for the first time, serves as a tool to demonstrate the validity and expediency of the multipole coupling method.

\subsection{Resonance energy transfer}

Resonance energy transfer $[1,18,20]$ is a spontaneous process which can be appropriately described within the quantum electrodynamical framework. It involves the migration of energy from an electronically excited donor, say centre $A$, to a nearby acceptor, $B$, residing in its ground state. Generally, centre $A$ is electronically distinct from centre $B$. Succinctly, the initial and final states of the process are given by

$$
\begin{gathered}
\mid \text { initial }\rangle=\left|E_{\alpha}^{A}, E_{0}^{B}\right\rangle, \\
\mid \text { final }\rangle=\left|E_{0}^{A}, E_{\beta}^{B}\right\rangle,
\end{gathered}
$$

where the energy transferred from donor $A$ to acceptor $B$ is given by $E_{\alpha 0}^{A}=E_{\beta 0}^{B}$. The interaction invokes the exchange of a virtual photon. Using the method set forth in section 3 , and by inspection of the relevant time-ordered diagram, figure 1, we can readily determine an expression for the quantum probability amplitude $M^{\text {RET }}$ connecting the initial and final quantum states of the system. Since there is no throughput radiation, it can easily be seen that the radiative factor in expression (11) is trivial and equals unity. In each of the centres, $A$ and $B$, only one photon event occurs; therefore the quantum amplitude $M^{\text {RET }}$ simply involves the transition dipoles for each centre, connected by the retarded potential tensor:

$$
M^{\mathrm{RET}}=\mu_{i}^{0 \alpha(A)} V_{i j}\left(\frac{E_{\alpha 0}^{A}}{\hbar c} ; \mathbf{R}\right) \mu_{j}^{\beta 0(\mathrm{~B})} .
$$

In this case, the most elementary intermolecular interaction, the coupling method is straightforward. It must be brought to attention that the interaction potential in expression (17) depends on the magnitude of an effective wave vector $\kappa$. Following the instructions in section 3 , this magnitude is 


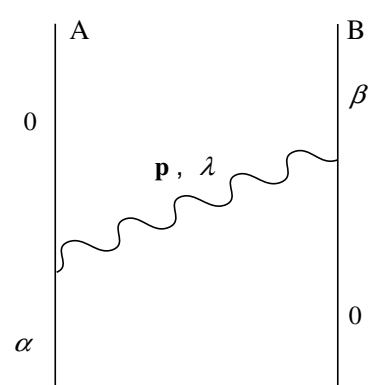

(a)

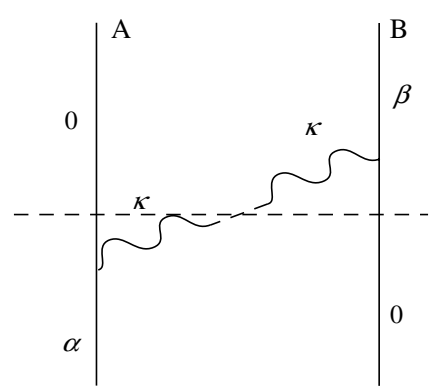

(b)
Figure 1. One of two time-ordered diagrams representing RET between two centres $A$ and $B ; \alpha$ and $\beta$ indicate the respective excited states. The verticals denote world lines of the two particles, wavy lines outside them denote real (laser) photons and those inside denote virtual photons; time progresses upwards. The corresponding ground state is represented by 0 : (a) traditional representation, the label $(\mathbf{p}, \lambda)$ indicating the transfer of a virtual photon; (b) process from the coupling method point of view, a simple contraction of two one-centre molecular tensors with a retarded potential linkage between them. The label $\kappa$ indicates the effective wave vector defined as $\kappa=E_{\alpha 0}^{A} / \hbar c$.

determined by the difference in energy between the initial and intermediate molecular states of both centres $A$ and $B$ :

$$
\begin{aligned}
\hbar c \kappa & \equiv\left|\left(E_{0}^{A}+E_{0}^{B}\right)-\left(E_{\alpha}^{A}+E_{0}^{B}\right)\right|=E_{\alpha 0}^{A} \\
& \equiv\left|\left(E_{\alpha}^{A}+E_{\beta}^{B}\right)-\left(E_{\alpha}^{A}+E_{0}^{B}\right)\right|=E_{\beta 0}^{B} .
\end{aligned}
$$

\subsection{Laser-assisted resonance energy transfer}

The process of laser-assisted resonance energy transfer (LARET) represents a development of RET, whereby the energy transfer is assisted by an auxiliary laser beam. The mechanism for the engagement of the throughput radiation entails additional photon events occurring at the donor and acceptor centres (see figure 2). The same net energy is transferred from one centre to the other as in RET. Defining the laser input as comprising $n$ photons with wave vector $\mathbf{k}$ and polarization $\lambda$, the initial and final states of the system can be written as

$$
\begin{gathered}
\mid \text { initial }\rangle=\left|E_{\alpha}^{A}, E_{0}^{B} ; n(\mathbf{k}, \lambda)\right\rangle, \\
\mid \text { final }\rangle=\left|E_{0}^{A}, E_{\beta}^{B} ; n(\mathbf{k}, \lambda)\right\rangle,
\end{gathered}
$$

where only the radiation state of the auxiliary beam, $|n(\mathbf{k}, \lambda)\rangle$, is explicitly represented. Here, the radiative factor (12) takes the simple form

$$
R_{\mathrm{rad}}=-\frac{n \hbar c k}{2 V \varepsilon_{o}} \exp [-\mathrm{ik} \cdot \mathbf{R}]
$$

Since LARET involves two real photon interactions in addition to the virtual photon coupling, two possibilities arise: in one case (labelled LARET $^{(1)}$ below) each centre undergoes two interactions - one with a real photon and the other a virtual photon; therefore, the molecular tensors in the transition matrix are two generalized polarizability tensors, these being linked through the retarded potential tensor $V_{j k}$. Again, two

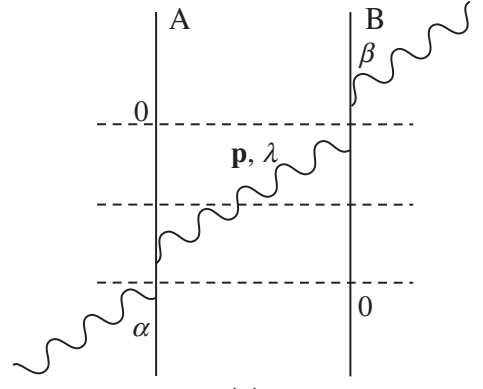

(a)

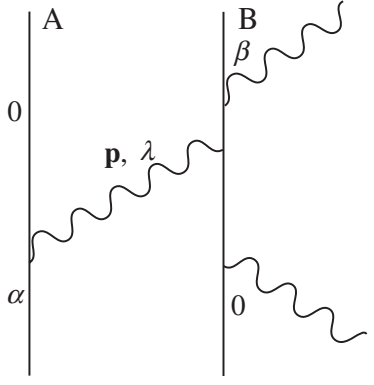

(b)
Figure 2. LARET process: in mechanism (a) the auxiliary beam interacts with both $A$ and $B$ and in mechanism (b) the auxiliary beam interacts with only one of the centres. In each case there are 24 different contributions and mirrored counterparts.

different possibilities emerge; the throughput radiation can result in the absorption of a real photon at centre $A$ and the emission of a real photon of the same mode at $B$, or vice versa. These two instances are reflected in the two contributions to the matrix element shown below:

$$
\begin{aligned}
& M^{\text {LARET(1) }}=-\frac{n \hbar c k}{2 V \varepsilon_{o}} \\
& \times\left[e_{i}^{\left(\lambda_{1}\right)}(\mathbf{k}) \alpha_{i j}^{0 \alpha(A)}\left(-k-\frac{E_{\alpha 0}^{A}}{\hbar c} ; k\right) V_{j k}\left(k+\frac{E_{\alpha 0}^{A}}{\hbar c} ; \mathbf{R}\right)\right. \\
& \quad \times \alpha_{k l}^{\beta 0(\mathrm{~B})}\left(-k ; k+\frac{E_{\alpha 0}^{A}}{\hbar c}\right) \bar{e}_{l}^{\left(\lambda_{1}\right)}(\mathbf{k}) \exp (-\mathrm{ik} \cdot \mathbf{R}) \\
& +\bar{e}_{i}^{\left(\lambda_{1}\right)}(\mathbf{k}) \alpha_{i j}^{0 \alpha(A)}\left(k-\frac{E_{\alpha 0}^{A}}{\hbar c} ;-k\right) V_{j k}\left(-k+\frac{E_{\alpha 0}^{A}}{\hbar c} ; \mathbf{R}\right) \\
& \left.\quad \times \alpha_{k l}^{\beta 0(\mathrm{~B})}\left(k ;-k+\frac{E_{\alpha 0}^{A}}{\hbar c}\right) e_{l}^{\left(\lambda_{1}\right)}(\mathbf{k}) \exp (\mathrm{ik} \cdot \mathbf{R})\right] .
\end{aligned}
$$

The result, in agreement with [45], involves the generalized polarizability defined by

$\alpha_{i j}^{m n(\xi)}\left( \pm k_{2} ; \pm k_{1}\right)=\sum_{s}\left[\frac{\mu_{i}^{m s(\xi)} \mu_{j}^{s n(\xi)}}{E_{s n}^{(\xi)} \mp \hbar c k_{2}}+\frac{\mu_{j}^{m s(\xi)} \mu_{i}^{s n(\xi)}}{E_{s n}^{(\xi)} \mp \hbar c k_{1}}\right]$,

as in [20] (p 113). In its application to the LARET process, however, the above tensor depends on the magnitude of effective wave vectors $\pm k$ and $\pm\left(k-\frac{E_{\alpha 0}^{A}}{\hbar c}\right)$.

To complete the description of LARET additional terms, based on static intermolecular interactions, must also be addressed. These LARET $^{(2)}$ contributions arise from a mechanism where both the photon events concerning the auxiliary beam occur at the same centre (the two centres still being coupled by a virtual photon). In the electricdipole approximation, this mechanism effectively involves the contraction of a transition dipole in, say, centre $A$, $\mu_{i}^{0 \alpha}$, with a transition hyperpolarizability tensor at centre $B$, $\beta_{i j l}^{\beta 0(\mathrm{~B})}\left(-k, \frac{E_{\alpha 0}^{A}}{\hbar c}, k\right)$, again linked through the retarded potential tensor, $V_{i j}\left(\frac{E_{\alpha 0}^{A}}{\hbar c} ; \mathbf{R}\right)$; the other possibility is that the roles of $A$ and $B$ are reversed. The total static contribution to the transition amplitude is then

$M^{\mathrm{LARET}(2)}=\frac{n \hbar c k}{2 \varepsilon_{o} V}\left[\bar{e}_{i}^{\left(\lambda_{1}\right)}(\mathbf{k}) \beta_{i j l}^{\beta 0(\mathrm{~B})}\left(-k, \frac{E_{\alpha 0}^{A}}{\hbar c}, k\right)\right.$ 


$$
\begin{aligned}
& \times V_{j k}\left(\frac{E_{\alpha 0}^{A}}{\hbar c} ; \mathbf{R}\right) \mu_{k}^{0 \alpha(A)} e_{l}^{\left(\lambda_{1}\right)}(\mathbf{k})+\bar{e}_{i}^{\left(\lambda_{1}\right)}(\mathbf{k}) \\
& \left.\times \beta_{i j l}^{0 \alpha(A)}\left(-k,-\frac{E_{\alpha 0}^{A}}{\hbar c}, k\right) V_{j k}\left(\frac{E_{\alpha 0}^{A}}{\hbar c} ; \mathbf{R}\right) \mu_{k}^{\beta 0(\mathrm{~B})} e_{l}^{\left(\lambda_{1}\right)}(\mathbf{k})\right]
\end{aligned}
$$

where the tensor $\beta_{i j l}^{f i(\xi)}$ is defined in its usual form [18]:

$$
\begin{aligned}
\beta_{j k l}^{m n(\xi)}\left(k_{3}, k_{2}, k_{1}\right) & \left\{\sum_{s, r} \frac{\mu_{j}^{m s(\xi)} \mu_{k}^{s r(\xi)} \mu_{l}^{r n(\xi)}}{\left(E_{s n}^{(\xi)}-\hbar c k_{2}-\hbar c k_{1}\right)\left(E_{r n}^{(\xi)}-\hbar c k_{1}\right)}\right. \\
& +\frac{\mu_{j}^{m s(\xi)} \mu_{l}^{s r(\xi)} \mu_{k}^{r n(\xi)}}{\left(E_{s n}^{(\xi)}-\hbar c k_{2}-\hbar c k_{1}\right)\left(E_{r n}^{(\xi)}-\hbar c k_{2}\right)} \\
& +\frac{\mu_{k}^{m s(\xi)} \mu_{j}^{s r(\xi)} \mu_{l}^{r n(\xi)}}{\left(E_{s n}^{(\xi)}-\hbar c k_{3}-\hbar c k_{1}\right)\left(E_{r n}^{(\xi)}-\hbar c k_{1}\right)} \\
& +\frac{\mu_{k}^{m s(\xi)} \mu_{l}^{s r(\xi)} \mu_{j}^{r n(\xi)}}{\left(E_{s n}^{(\xi)}-\hbar c k_{3}-\hbar c k_{1}\right)\left(E_{r n}^{(\xi)}-\hbar c k_{3}\right)} \\
& +\frac{\mu_{l}^{m s(\xi)} \mu_{j}^{s r(\xi)} \mu_{k}^{r n(\xi)}}{\left(E_{s n}^{(\xi)}-\hbar c k_{3}-\hbar c k_{2}\right)\left(E_{r n}^{(\xi)}-\hbar c k_{2}\right)} \\
& \left.+\frac{\mu_{l}^{m s(\xi)} \mu_{k}^{s r(\xi)} \mu_{j}^{r n(\xi)}}{\left(E_{s n}^{(\xi)}-\hbar c k_{3}-\hbar c k_{2}\right)\left(E_{r n}^{(\xi)}-\hbar c k_{3}\right)}\right\} .
\end{aligned}
$$

The contributions given by expressions (21) and (23) are in accordance with previous work, based on the traditional methods [18, 45]. Figure 3 shows a particular time-ordered diagram for each type of contribution, one dynamic and the other static. In figure 3(a) it can be seen that in the dynamic case the contribution to the transition matrix for LARET involves the contraction of the radiative tensor with two polarizability tensors, $\alpha_{i j}^{\xi}\left(\kappa, \kappa^{\prime}\right)$, one for each centre. These are linked via the retarded coupling tensor $V_{j k}(\kappa, R)$. Similarly, from figure 3(b), it can be interpreted that the static case involves the contraction of the radiative tensor with a hyperpolarizability tensor in one centre, which in turn is linked to a static transition dipole $\mu_{k}^{r s}$ via the retarded coupling tensor $V_{j k}(\kappa, R)$.

\subsection{RET in the vicinity of a third centre}

Here, the effects on the rate of RET between a pair of centres, $A$ and $B$, due to the presence of a third centre, $C$, are analysed [24]. If such a third-party centre $C$ is polar, its presence may result in a significant variation of the transfer rate. Furthermore, the presence of such a body can effectively switch on the RET process in cases where such energy transfer is forbidden due to orientational effects, or on symmetry grounds. In general, the initial and final molecular states for this system are

$$
\begin{gathered}
\mid \text { initial }\rangle=\left|E_{\alpha}^{A}, E_{0}^{B}, E_{0}^{C}\right\rangle, \\
\mid \text { final }\rangle=\left|E_{0}^{A}, E_{\beta}^{B}, E_{0}^{C}\right\rangle .
\end{gathered}
$$

The electromagnetic coupling between the three species is solely mediated by virtual photons. Apart from the

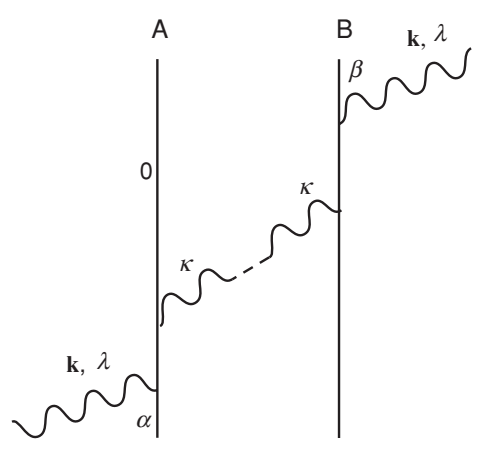

(a)

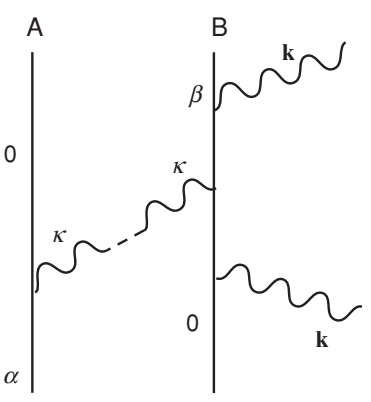

(b)
Figure 3. (a) Here the process contribution can be clearly seen to involve a product of two polarizability tensors, one consisting of the absorption of a photon of energy $\hbar c k$ and the emission of a photon of energy $\hbar c \kappa=\hbar c k+E_{\alpha 0}^{A}$. (b) As in the previous figure, the static contribution can be seen to involve the product of the static dipole moment with the hyperpolarizability tensor.

conventional operation of RET which does not involve $C$, there are now three possible mechanisms associated with threecentre RET. Each one has either centre $A, B$ or $C$ acting as an electronic bridge between the remaining two. As a consequence of the time-energy uncertainty principle, there are 72 possible time orderings associated with this process. Even if the state-sequence method is used, where all time orderings can be depicted in a single diagram, the calculational expense is considerable (for more details of this approach see [24]). Nevertheless, inspecting figure 4 and using the coupling method outlined in section 3 , the transition matrix for the threebody RET process can be obtained much more directly and straightforwardly. Using the algorithm described in section 3, the result emerges as follows:

$$
\begin{aligned}
& \mu_{i}^{00(C)} V_{i j}\left(0 ; \mathbf{R}_{A C}\right) \alpha_{j k}^{0 \alpha(A)}\left(-\frac{E_{\alpha 0}^{A}}{\hbar c}, 0\right) V_{k l}\left(\frac{E_{\alpha 0}^{A}}{\hbar c} ; \mathbf{R}_{B A}\right) \mu_{l}^{\beta 0(\mathrm{~B})} \\
& +\mu_{i}^{0 \alpha(A)} V_{i j}\left(\frac{E_{\alpha 0}^{A}}{\hbar c} ; \mathbf{R}_{B A}\right) \alpha_{j k}^{\beta 0(\mathrm{~B})}\left(0, \frac{E_{\alpha 0}^{A}}{\hbar c}\right) V_{k l}\left(0 ; \mathbf{R}_{C B}\right) \mu_{l}^{00(C)} \\
& +\mu_{i}^{0 \alpha(A)} V_{i j}\left(\frac{E_{\alpha 0}^{A}}{\hbar c} ; \mathbf{R}_{C A}\right) \alpha_{j k}^{00(C)}\left(-\frac{E_{\alpha 0}^{A}}{\hbar c},+\frac{E_{\alpha 0}^{A}}{\hbar c}\right) \\
& \quad \times V_{k l}\left(\frac{E_{\alpha 0}^{A}}{\hbar c} ; \mathbf{R}_{B C}\right) \mu_{l}^{\beta 0(\mathrm{~B})}
\end{aligned}
$$

As with cases studied previously, care must be taken when considering the effective wave vectors $\kappa_{i}$ in both the polarizability tensor $\alpha_{j k}^{(\xi)}\left(\kappa_{1}, \kappa_{2}\right)$ and the coupling tensor $V_{k l}\left(\kappa_{3}, \mathbf{R}_{\xi \xi^{\prime}}\right)$. In this respect each of the three mechanisms must be regarded separately. This example reflects the advantages that the coupling method presents, where by simple inspection of only one time-ordered diagram for each of the mechanisms involved in the optical process, a complete expression for the transition matrix can be obtained.

\subsection{Optical binding: two-, three- and N-body cases}

Optical binding is an optomechanical effect exhibited by systems of micro- and nano-particles, suitably irradiated with 


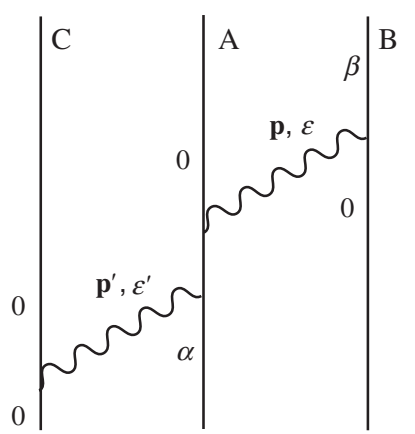

(a)

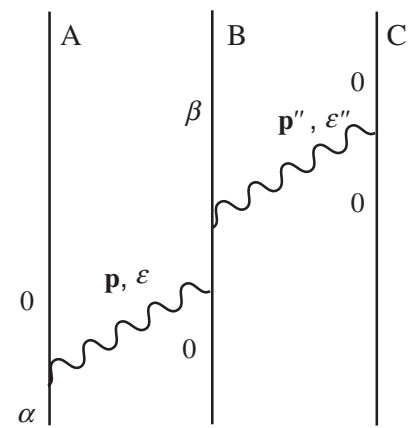

(b)

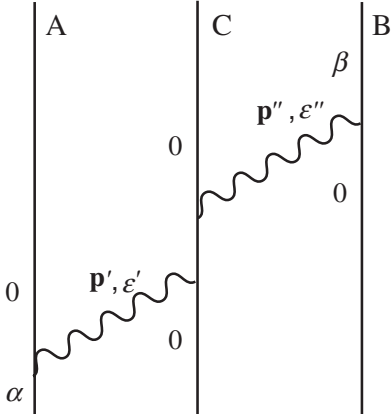

(c)

Figure 4. Third body influence to RET. (a), (b) and (c) each depict one of the possible 24 time-ordered contributions for each mechanism: (a) static dipole of $C$ interacts with donor $A$; (b) static dipole of $C$ interacts with donor $B$;(c) static dipole of $C$ acts as bridging species between the donor and acceptor.

off-resonance laser light [22, 46, 47]. Physically distinct from standing-wave and other forms of holographic optical trap, the phenomenon arises as a result of an inter-particle coupling with individual radiation modes, leading to optically induced modifications to Casmir-Polder interactions. These optically induced inter-particle interactions give rise to forces and torques (usually described as optical binding, although the forces are not necessarily attractive in form) and they have been of a particular focus in recent extensive investigations (see for example [2, 39-41, 48-52] and references therein). The phenomenon has increasingly been advocated as a tool for optical manipulation and configuration of particles, and many optically induced arrays have been observed experimentally [52-54].

The description of optical binding within molecular QED is mathematically similar to that given for LARET. Starting with two centres, $A$ and $B$, in the presence of a laser beam, the 'initial' and 'final' states of the system can be written as

$$
\begin{gathered}
\mid \text { initial }\rangle=\left|E_{0}^{A}, E_{0}^{B} ; n(\mathbf{k}, \lambda)\right\rangle, \\
\mid \text { final }\rangle=\left|E_{0}^{A}, E_{0}^{B} ; n(\mathbf{k}, \lambda)\right\rangle .
\end{gathered}
$$

Here the equivalence of the two system state vectors signifies that diagonal elements of the transition matrix are to be derived-including non-trivial correction terms that arise from a coupling of off-diagonal matrix elements. The result is, therefore, to be interpreted as an energy (shift). As can be seen from expression (27), the particles $A$ and $B$ are

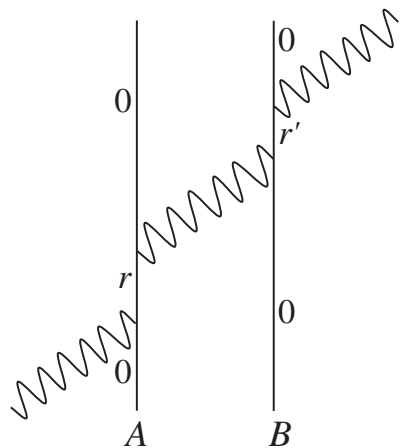

(a)

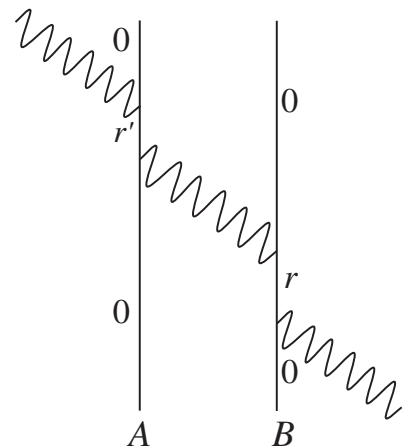

(b)
Figure 5. Two typical Feynman diagrams (each with 23 further permutations) for the calculation of dynamic contributions to the laser-induced interaction energy. Adapted from [46]. The static contribution, not shown here, corresponds to the real photon being scattered at one centre only (see figure 3(b)).

generally considered to be in their lowest energy state, i.e. their ground state, and remain in these states beyond any interaction with the highly intense throughput radiation. Four possible mechanisms must be considered, as shown in figure 5. These are as follows: (a) the case where a photon absorption event occurs in centre $A$, and a photon emission event, of the same mode, occurs at centre $B$; (b) the second mechanism comprises the mirror case where the roles of $A$ and $B$ are exchanged; (c) and (d) the cases which involve the static form of contribution. Based on the coupling method described in section 3 , the resulting energy shift between the interacting centres due to all four mechanisms is

$$
\begin{aligned}
& \Delta E(\mathbf{k}, \mathbf{R})=\left(\frac{I}{\varepsilon_{0} c}\right) e_{i}^{(\lambda)} \bar{e}_{l}^{(\lambda)} \operatorname{Re}\left\{\alpha_{i j}^{A}(-k, k) V_{j k}(k, \mathbf{R})\right. \\
& \quad \times \alpha_{k l}^{B}(-k, k) \exp (-\mathrm{ik} \cdot \mathbf{R})+\alpha_{i j}^{B}(-k, k) V_{j k}(k, \mathbf{R}) \\
& \quad \times \alpha_{k l}^{A}(-k, k) \exp (\mathrm{ik} \cdot \mathbf{R})+\frac{1}{2} V_{j k}(0, \mathbf{R})\left[\beta_{i j l}^{A}(-k, 0, k) \mu_{k}^{B}\right. \\
& \left.\left.\quad+\mu_{k}^{A} \beta_{i j l}^{B}(-k, 0, k)\right]\right\},
\end{aligned}
$$

in accordance with previous results [18, 40, 41]. From expression (28) it can be observed that the energy shift for optical binding is in a certain sense simply a particular case of the LARET result, in which $E_{\alpha 0}^{A}=E_{\beta 0}^{B} \equiv 0$, i.e. the molecular states $\alpha$ and $\beta$ are replaced by the ground state [47, 50, 51].

Based on the analysis of expression (28), assuming two spherical particles, the spatial dependence of the optical energy reveals richly detailed potential energy landscapes, which can be exhibited as a function of the separation vector $\mathbf{R}$. It is apparent that these principles afford a tractable basis for extension to a system of $N$ spherical particles, irradiated with a linearly polarized beam. In general, the determination of the optically induced potential experienced by the whole assembly requires the evaluation of equation (28) for $\frac{1}{2} N(N-1)$ particle pairs (obviously making depictions of the system significantly more complex when considering a large number of particles).

Beyond the particle pair approximation for an $N$-particle system, further contributions to the optically induced energy shift could be calculated from first principles based on either 


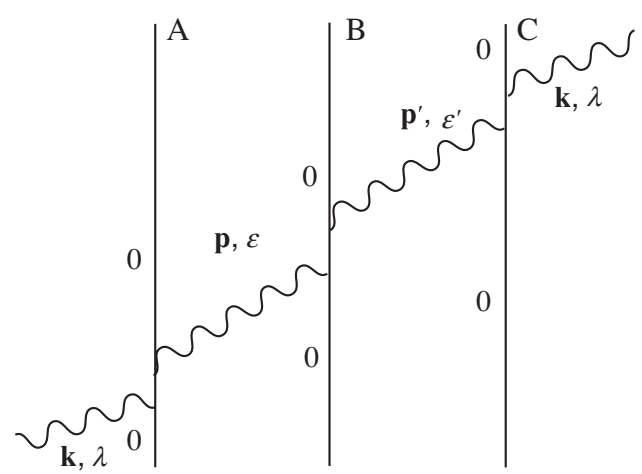

Figure 6. Optical binding for three particles. Here we show one of the 720 possible time-ordered diagrams.

the time-ordered diagrams or the state-sequence method. A system involving $N$ particles would entertain $N$ ! mechanisms, each involving $(2 N)$ ! time-ordered diagrams-for example, in the case of a three-particle system this would involve consideration of six different mechanisms each involving 720 time orderings, figure 6 being one of the possible orderings. Fortunately the coupling method offers a straightforward answer to such a problem. This method can deliver a result for a system with an unspecified number of particles, $N$. The energy shift for such a system can readily be identified as the sum of all possible contributions of the general form

$$
\begin{aligned}
& \Delta E^{\left\{\xi_{1}, \ldots \xi_{N}\right\}}=\frac{n \hbar c k}{2 V \varepsilon_{o}} e_{i_{0}}^{(\lambda)}(\mathbf{k}) \bar{e}_{i_{N}}^{(\lambda)}(\mathbf{k}) \\
& \quad \times \alpha_{i_{0} i_{1}}^{\left(\xi_{1}\right)}(-k ; k) \prod_{p=1}^{N-1}\left[V_{i_{p} i_{p+1}}\left(k, \mathbf{R}_{\xi_{p} \xi_{p+1}}\right) \alpha_{i_{p+1} i_{p+2}}^{\left(\xi_{p+1}\right)}(-k ; k)\right] \\
& \quad \times \exp \left(-\mathrm{i} \mathbf{k} \cdot \mathbf{R}_{\xi_{N} \xi_{1}}\right) .
\end{aligned}
$$

Here, the set of superscript labels $\left\{\xi_{1}, \ldots, \xi_{N}\right\}$ indicates that all possible permutations of $\xi_{i}$ must be taken into account.

In particular, for the simplest case of a three-centre system, first studied in [8], we have contributions of the form

$$
\begin{aligned}
\Delta E^{\{A, B, C\}}=\frac{n \hbar c k}{2 V \varepsilon_{o}} e_{i_{0}}^{(\lambda)}(\mathbf{k}) \bar{e}_{i_{N}}^{(\lambda)}(\mathbf{k}) \\
\quad \times \alpha_{i_{0} i_{1}}^{(A)}(-k ; k) V_{i_{1} i_{2}}\left(k, \mathbf{R}_{B A}\right) \alpha_{i_{2} i_{3}}^{(B)}(-k ; k) V_{i_{3} i_{4}}\left(k, \mathbf{R}_{C B}\right) \\
\quad \times \alpha_{i_{4} i_{5}}^{(C)}(-k ; k) \exp \left(-\mathrm{i} \mathbf{k} \cdot \mathbf{R}_{C A}\right) .
\end{aligned}
$$

Accommodating the other five permutations of $\{A, B, C\}$, the net results are as follows:

$$
\begin{aligned}
\Delta E & =\frac{n \hbar c k}{2 V \varepsilon_{o}} e_{i_{0}}^{(\lambda)}(\mathbf{k}) \bar{e}_{i_{N}}^{(\lambda)}(\mathbf{k}) \\
& \times\left[\alpha_{i_{0} i_{1}}^{(A)}(-k ; k) V_{i_{1} i_{2}}\left(k, \mathbf{R}_{B A}\right) \alpha_{i_{2} i_{3}}^{(B)}(-k ; k) V_{i_{3} i_{4}}\left(k, \mathbf{R}_{C B}\right)\right. \\
& \times \alpha_{i_{4} i_{5}}^{(C)}(-k ; k) \cos \left(\mathbf{k} \cdot \mathbf{R}_{C A}\right)+\alpha_{i_{0} i_{1}}^{(A)}(-k ; k) V_{i_{1} i_{2}}\left(k, \mathbf{R}_{B A}\right) \\
& \times \alpha_{i_{2} i_{3}}^{(C)}(-k ; k) V_{i_{3} i_{4}}\left(k, \mathbf{R}_{C B}\right) \alpha_{i_{4} i_{5}}^{(B)}(-k ; k) \cos \left(\mathbf{k} \cdot \mathbf{R}_{B A}\right) \\
& +\alpha_{i_{0} i_{1}}^{(B)}(-k ; k) V_{i_{1} i_{2}}\left(k, \mathbf{R}_{B A}\right) \alpha_{i_{2} i_{3}}^{(A)}(-k ; k) V_{i_{3} i_{4}}\left(k, \mathbf{R}_{C B}\right) \\
& \left.\times \alpha_{i_{4} i_{5}}^{(C)}(-k ; k) \cos \left(\mathbf{k} \cdot \mathbf{R}_{C B}\right)\right] .
\end{aligned}
$$

Expression (31) perfectly illustrates the advantages that use of the propagator method offers, where a result for the energy shift can be effortlessly obtained from a seemingly complicated process that would otherwise be calculationally costly.

Finally, it is worth observing how to extend the results to larger particles. Experimentally, optical binding is generally encountered between nano-particles, optical centres or molecular aggregates formed by a certain number of molecules, $p$. To consider such systems we need to only entertain an effective polarizability given by

$$
\chi_{i j}^{(\zeta)}=\sum_{\xi}^{p} \alpha_{\lambda \mu}^{(\xi)} I_{i \lambda}^{(\xi)} I_{j \mu}^{(\xi)} \exp \left(\mathbf{i k} \cdot \Delta \mathbf{R}_{\xi}\right),
$$

where the $I_{\lambda i}^{(\xi)}$ are the cosines of the angles between the particle-fixed and molecule-fixed axes. The relative position of each molecule $\xi$ within a particular aggregate $\zeta$ is given by $\Delta \mathbf{R}_{\xi}$. Such aggregates can be considered as mesoscopically disordered materials, within which local domains possess particular structures. For a more detailed analysis see [8].

\section{Discussion}

The different and varied intermolecular processes reviewed in section 4 clearly show the advantages that the retarded coupling method represents. It is a concise approach to the analysis and study of a host of optical processes. Not only are time-consuming calculations circumvented, but also it is possible to draw mathematical connections and similarities between different processes, such as the case with LARET and optical binding.

In this work we have restricted consideration to the electric-dipole approximation. Nevertheless, extending the coupling method to incorporate higher order multipole coupling should be straightforward. Processes where the intermolecular interactions involve more than one virtual photon, such as the case of the Casimir-Polder dispersion interactions [21], need further consideration. The basis for such interactions can still be regarded as described by the general expression (11); however, the retarded potential tensor given by (5) is no longer applicable, because it does not allow for any temporal cross-over between two virtual photons (see [21]). If a suitable bridging potential tensor were to be determined, then the extension of the retarded coupling approach to include dispersion interaction processes should be possible. This represents rich ground for future work.

\section{Acknowledgment}

This work is funded by the Engineering and Physical Sciences Research Council.

\section{References}

[1] Andrews D L and Demidov A A 1999 Resonance Energy Transfer (Chichester: Wiley)

[2] Guillon M, Moine O and Stout B 2006 Longitudinal optical binding of high optical contrast microdroplets in air Phys. Rev. Lett. 96143902 
[3] Sapsford K E, Berti L and Medintz I L 2006 Materials for fluorescence resonance energy transfer analysis: beyond traditional donor-acceptor combinations Angew. Chem., Int Ed. 45 4562-88

[4] Saini S, Singh H and Bagchi B 2006 Fluorescence resonance energy transfer (FRET) in chemistry and biology: non-Forster distance dependence of the FRET rate $J$. Chem. Sci. 118 23-35

[5] Metzger N K, Marchington R F, Mazilu M, Smith R L Dholakia K and Wright E M 2007 Measurement of the restoring forces acting on two optically bound particles from normal mode correlations Phys. Rev. Lett. 98 068102-4

[6] Chen F, Klimchitskaya G L, Mostepanenko V M and Mohideen U 2007 Control of the Casimir force by the modification of dielectric properties with light Phys. Rev. B 76035338

[7] Passante R, Persico F and Rizzuto L 2007 Nonlocal field correlations and dynamical Casimir-Polder forces between one excited- and two ground-state atoms J. Phys. B: At. Mol. Opt. Phys. 40 1863-74

[8] Rodríguez J, Dávila Romero L C and Andrews D L 2008 Optical binding in nanoparticle assembly: Potential energy landscapes Phys. Rev. A 78 043805-7

[9] Andrews D L and Rodríguez J 2008 Collapse of optical binding under secondary irradiation Opt. Lett. 33 1830-2

[10] Avery J S 1966 Resonance energy transfer and spontaneous photon emission Proc. Phys. Soc. London 88 1-8

[11] Gomberoff L and Power E A 1966 Resonance transfer of excitation Proc. Phys. Soc. London 88 281-4

[12] Salam A 2005 A general formula for the rate of resonant transfer of energy between two electric multipole moments of arbitrary order using molecular quantum electrodynamics J. Chem. Phys. 122044112

[13] Bradshaw D S, Leeder J M, Rodríguez J and Andrews D L 2008 Resonance energy transfer and interface forces: quantum electrodynamical analysis Complex Light and Optical Forces II (San Jose, CA: SPIE) 6905690503

[14] Andrews D L, Bradshaw D S, Leeder J M and Rodríguez J 2008 Dynamics of the dispersion interaction in an energy transfer system Phys. Chem. Chem. Phys. 10 5250-5

[15] Andrews D L and Leeder J M 2009 On the interactions between molecules in an off-resonant laser beam: evaluating the response to energy migration and optically induced pair forces J. Chem. Phys. 130034504

[16] Thirunamachandran T 1980 Intermolecular interactions in the presence of an intense radiation-field Mol. Phys. $40393-9$

[17] Burns M M, Fournier J M and Golovchenko J A 1989 Opti. Binding Phys. Rev. Lett. 63 1233-6

[18] Allcock P, Jenkins R D and Andrews D L 2000 Laser-assisted resonance-energy transfer Phys. Rev. A 61023812

[19] Power E A and Thirunamachandran T 1993 A new insight into the mechanism of intermolecular forces Chem. Phys. 171 1-7

[20] Craig D P and Thirunamachandran T 1998 Molecular Quantum Electrodynamics: An Introduction to Radiation-Molecule Interactions (Mineola, NY: Dover)

[21] Alligood B W and Salam A 2007 On the application of state sequence diagrams to the calculation of the Casimir-Polder potential Mol. Phys. 105 395-404

[22] Bradshaw D S and Andrews D L 2005 Optically induced forces and torques: interactions between nanoparticles in a laser beam Phys. Rev. A 72033816

[23] Dávila Romero L C, Rodríguez J and Andrews D L 2008 Optically induced multi-particle structures: multi-dimensional energy landscapes Nanophotonics II ed D L Andrews (Strasbourg, France: SPIE) 6988 69880L

[24] Daniels G J and Andrews D L 2002 The electronic influence of a third body on resonance energy transfer J. Chem. Phys. 116 6701-12
Daniels G J and Andrews D L 2002 The electronic influence of a third body on resonance energy transfer J. Chem. Phys. 117 6882-93 (erratum)

[25] Jenkins R D, Andrews D L and Dávila Romero L C 2002 A new diagrammatic methodology for non-relativistic quantum electrodynamics J. Phys. B: At. Mol. Opt. Phys. 35 445-68

[26] Casimir H B G and Polder D 1946 Influence of retardation on the London-Van der Waals forces Nature 158 787-8

[27] Casimir H B G and Polder D 1948 The influence of retardation on the London-Van der Waals forces Phys. Rev. 73 360-72

[28] Woolley R G 2000 Gauge invariance in non-relativistic electrodynamics Proc. $R$. Soc A 456 1803-19

[29] Woolley R G 2001 Light scattering, origin invariant multipole moments and molecular property tensors: the case of electric-field-gradient-induced birefringence Mol. Phys. 99 547-58

[30] Gray C G and Gubbins K E 1984 Theory of Molecular Fluids (Oxford: Clarendon)

[31] Rzazewski K and Boyd R W 2004 Equivalence of interaction Hamiltonians in the electric dipole approximation J. Mod. Opt. 51 1137-47

[32] Scholes G D and Andrews D L 1997 Damping and higher multipole effects in the quantum electrodynamical model for electronic energy transfer in the condensed phase J. Chem. Phys. 107 5374-84

[33] Salam A 2005 Generalized expressions for resonant excitation transfer and retarded dispersion energy shifts obtained using multipolar quantum electrodynamics Int. J. Quantum Chem. $105762-6$

[34] Passante R and Persico F 1999 Virtual photons and three-body forces J. Phys. B: At. Mol. Opt. Phys. 32 19-25

[35] Juzeliūnas G and Andrews D L 2000 Quantum electrodynamics of resonance energy transfer $A d v$. Chem. Phys. 112 357-410

[36] Andrews D L and Bradshaw D S 2004 Virtual photons, dipole fields and energy transfer: a quantum electrodynamical approach Eur. J. Phys. 25 845-58

[37] Power E A and Thirunamachandran T 1993 Casimir-Polder potentials as an interaction between induced dipoles Phys. Rev. A 48 4761-3

[38] Craig D P and Thirunamachandran T 1999 New approaches to chiral discrimination in coupling between molecules Theor. Chem. Acc. 102 112-20

[39] Salam A 2006 On the effect of a radiation field in modifying the intermolecular interaction between two chiral molecules J. Chem. Phys. 124014302

[40] Salam A 2006 Intermolecular interactions in a radiation field via the method of induced moments Phys. Rev. A 73013406

[41] Salam A 2007 Two alternative derivations of the static contribution to the radiation-induced intermolecular energy shift Phys. Rev. A 76063402

[42] Salam A 2006 A general formula obtained from induced moments for the retarded van der Waals dispersion energy shift between two molecules with arbitrary electric multipole polarizabilities: I. Ground state interactions J. Phys. B: At. Mol. Opt. Phys. 39 S651-61

[43] Salam A 2006 A general formula obtained from induced moments for the retarded van der Waals dispersion energy shift between two molecules with arbitrary electric multipole polarizabilities: II. Excited state interactions J. Phys. B: At. Mol. Opt. Phys. 39 S663-9

[44] Naguleswaran S and Stedman G E 1996 Time reversal selection rules and gauge invariance in nonlinear optics J. Phys. B: At. Mol. Opt. Phys. 29 4027-40

[45] Bradshaw D S and Andrews D L 2008 Optically controlled resonance energy transfer: mechanism and configuration for all-optical switching J. Chem. Phys. 128144506 
[46] Bradshaw D S and Andrews D L 2005 Optically induced forces and torques: interactions between nanoparticles in a laser beam Phys. Rev. A 72033816

Bradshaw D S and Andrews D L 2006 Optically induced forces and torques: interactions between nanoparticles in a laser beam Phys. Rev. A 73039903 (erratum)

[47] Dávila Romero L C and Andrews D L 2008 Structured Light and Its Applications: An Introduction to Phase-Structured Beams and Nanoscale Optical Forces ed D L Andrews (Burlington, MA: Academic) pp 79-105

[48] Andrews D L, Crisp R G and Bradshaw D S 2006 Optically induced inter-particle forces: from the bonding of dimers to optical electrostriction in molecular solids J. Phys. B: At. Mol. Opt. Phys. 39 S637-50

[49] Grzegorczyk T M, Kemp B A and Kong J A 2006 Stable optical trapping based on optical binding forces Phys. Rev. Lett. 96113903
[50] Dávila Romero L C, Rodríguez J and Andrews D L 2008 Electrodynamic mechanism and array stability in optical binding Opt. Commun. 281 865-70

[51] Rodríguez J, Dávila Romero L C and Andrews D L 2008 Optical binding: potential energy landscapes and QED Complex Light and Optical Forces II ed D L Andrews (San Jose, CA: SPIE) $690569050 \mathrm{H}$

[52] Marchington R F, Mazilu M, Kuriakose S, Garces-Chavez V, Reece P J, Krauss T F, Gu M and Dholakia K 2008 Optical deflection and sorting of microparticles in a near-field optical geometry Opt. Exp. 16 3712-26

[53] Mohanty S K, Andrews J T and Gupta P K 2004 Optical binding between dielectric particles Opt. Exp. 12 2746-53

[54] Mellor C D, Fennerty T A and Bain C D 2006 Polarization effects in optically bound particle arrays Opt. Exp. 14 10079-88 